\title{
Macromolecular Type 1 Creatine Kinase to Total Creatine Kinase Ratio Measurement
}

National Cancer Institute

\section{Source}

National Cancer Institute. Macromolecular Type 1 Creatine Kinase to Total Creatine Kinase Ratio Measurement. NCI Thesaurus. Code C147319.

The determination of the ratio of macromolecular type 1 creatine kinase compared to total creatine kinase present in a sample. The measurement may be expressed as a ratio or percentage. 